\title{
Health Impact Assessment of the Proposed Opioid Prior Authorization Policy in the State of Utah, USA
}

\section{Abstract}

Background: From 2000-2014, Utah experienced a 400\% increase in opioidrelated deaths and currently ranks $4^{\text {th }}$ in the nation for such deaths. To address this problem, a policy was recently proposed by the Utah State Legislature that would require insurance prior authorization (PA) for certain high dose, long-acting opioid prescriptions. The purpose of this study is to analyze potential health impacts of the policy and to determine whether it would be an effective step in combatting Utah's opioid problem.

Methods: A rapid Health Impact Assessment (HIA) was performed to assess the potential health impacts of the proposed policy. Hypotheses were generated and a causal pathway was developed. Quantitative and qualitative data were gathered to assess each hypothesis and determine the direction, magnitude, likelihood, and distribution of each potential health impact.

Results: Several positive and negative health impacts of the policy were examined. Negative health impacts that are likely include increased time and cost for prescribers, and increased patient stress and anxiety due to difficulties in filling prescriptions. Positive health impacts include a modest decrease in the amount of opioids prescribed and a modest decrease in opioid consumption, leading to a decrease in the number of persons addicted to prescription opioids.

Conclusion: Given the potential for the proposed PA policy to decrease the number of opioid addicts, it is recommended that the Utah State Legislature move forward with the proposed policy. However, in order to mitigate potential negative health effects, it is recommended that pain specialists be exempt from opioid-related PA.

Keywords: Health Impact Assessment; Prior Authorization; Prescription opioids; Controlled substances; Legislative policy

Received: December 27, 2016, Accepted: January 07, 2017 Published: January 12, 2017

\section{Introduction and Background}

Utah, like most of the United States, is experiencing an opioid abuse and overdose epidemic. Utah experienced a $400 \%$ increase in opioid misuse and abuse deaths from 2000-2014. From 20122014, Utah was ranked $4^{\text {th }}$ in the nation for drug poisoning deaths, most of which were attributed to opioid overdose [1]. Increases in opioid prescription mirror increases in overdoses [2]. Opioid prescriptions nationally have jumped from 76 million in 1991 to 207 million in 2013, and 32\% of people aged 18 and older were prescribed an opioid pain medication in Utah in 2014 [2]. A recent study showed that $91 \%$ of prescription opioid overdose

\section{Emarie L. Covey' ${ }^{1}$, Lindsay M. Belvedere', Michael D. Barnes ${ }^{2}$}

1 MPHc, BSc, Department of Health Science, Brigham Young University, Provo, Utah, USA

$2 \mathrm{PhD}, \mathrm{MS}$, Department of Health Science, Brigham Young University, Provo, Utah, USA

Corresponding author: Michael D. Barnes

झ michael_barnes@byu.edu

Department of Health Science, 4103 LSB, Brigham Young University, Provo, Utah, 84602, USA.

Citation: Covey EL, Belvedere LM, Barnes MD. Health Impact Assessment of the Proposed Opioid Prior Authorization Policy in the State of Utah, USA. Health Syst Policy Res. 2017, 4:1. decedents in Utah obtained opioids from a health care provider, but others also received or stole unused prescription opioids from friends and family [3]. Thus, one key method to alleviating the opioid epidemic is curbing the overprescribing of opioids.

In order to prevent future opioid addiction and overdoses by restricting overprescribing, Representative Ray Ward (R), Utah State Legislature House District 19, recently proposed an opioid prior authorization (hereafter referred to as PA) bill [46]. PA requires insurance companies to pre-approve certain medications or procedures before reimbursing physicians for services or paying for medications. The bill, if passed, would 
require PA for any opioid prescription greater than 90 morphine equivalents; anytime benzodiazepines are prescribed to patients already taking opioids; and for any new patient starting chronic opioids (i.e. prescriptions longer than 10 days in duration) [4$6]$. Patients under hospice or other end-of-life care and surgical or injured patients would not be affected. The purpose of this study is to analyze the potential health impacts of the proposed policy and to determine whether or not it would be an effective step in combatting Utah's opioid problem. Other states may also learn helpful lessons from this report as they consider the health impacts of opioid PA. There is currently little academic research on opioid PA policies or how PA affects patient care and outcomes; this paper seeks to add to these understudied topics.

To accomplish the purpose of this study, a rapid Health Impact Assessment (hereafter referred to as HIA) was performed. This type of impact assessment focuses on the potential health effects of a proposed plan, project, or policy prior to its implementation. In general, HIAs highlight potential positive and negative health impacts of proposed plans, projects, or policies, which can be considered as part of the decision-making process. HIAs also make recommendations to maximize positive health impacts and minimize negative health impacts [7].

\section{Methods and Data Sources}

The six major steps of HIAs include a screening phase, scoping phase, assessment phase, recommendations phase, reporting phase and monitoring and evaluation phase [7]. This HIA reports the first four phases, which are described below.

\section{Screening Phase}

The HIA process began with a screening phase, which determined whether or not to conduct the HIA based on its potential, feasibility, and value in the decision-making process. As part of the screening phase, key stakeholders were identified and their willingness to participate in the HIA process was determined. Potential health effects and the availability of data to identify those health effects were determined. Given the availability of data, the likelihood of stakeholder participation, and most importantly, the timeliness of the results for legislative decision makers, it was decided that the HIA should be completed.

\section{Scoping Phase}

Next, the scoping phase aided in the planning of the HIA, and identified and prioritized the potential significant health impacts. Most of the scoping phase of this HIA included hypothesis generation and the development of a causal pathway (Figure 1), which displays the processes leading from the proposed policy to the likely health outcomes resulting from this policy. Each branch of the pathway represents a separate hypothesis that was explored in the assessment phase of this HIA.

\section{Assessment Phase}

The assessment phase of the HIA consisted of an analysis of the hypothesized health impacts proposed in the causal pathway (Figure 1) on affected populations, namely patients already using prescription opioids, patients who may need prescription opioids in the future, and their prescribers. Two types of data were collected as part of this HIA: 1) quantitative and qualitative data from literature review, and 2) qualitative data through stakeholder interviews. Stakeholder interviews were conducted with local individuals who were most likely to be interested in the outcome of the proposed policy, including physicians, dentists, pharmacists, and health department officials; interviews were also conducted with a health economist, a detective who frequently encounters illegal drug users, and a drug treatment center counselor. Together, gathered quantitative and qualitative data were assessed for each hypothesis. Following data assessment, the research team characterized the potential health impacts. The characterizations were compiled into the Summary of findings Table 1 under the criteria of direction, magnitude, likelihood, distribution, and certainty of evidence of each of the health impacts analyzed after the HIA team reached a consensus of each criteria.

\section{Recommendations Phase}

Recommendations were developed in response to the health impacts characterized in the assessment phase. Specific actions to avoid or minimize adverse health effects and maximize potential health benefits are discussed, and policy alternatives are considered.

\section{HIA Results}

The main causal pathways studied and reported in this paper include impacts to prescribers, impacts to pill availability, and impacts to patients. Each causal pathway contains hypotheses which were analyzed using stakeholder data and literature review findings.

\section{Impacts to Prescribers}

Changes in costs to prescribers After interviewing several prescribers, it was concluded that requiring PA for prescription opioids would greatly impact the practice of prescribers. Prescribers reported that PA requirements, no matter what they are for, are a significant burden, hassle, and cost for medical practitioners and pharmacists. This is largely due to having to hire extra staff to complete Pas [8-13]. Studies confirm these prescriber sentiments that PA requirements increase doctor time, hassle, paperwork, and cost [14, 15]. Furthermore, the concern was raised that increased paperwork for doctors results in sacrificing face-to-face time with patients, leading to poorer quality of care and poorer health outcomes for patients [8]. It was also noted that such a policy could be particularly problematic for pain clinics [8]. Pain specialists write many prescriptions for highdose opioids that would require PA under this policy; as such, the implementation of this policy may be a great burden to pain specialists, due to increased costs and paperwork [8].

Changes in prescribing habits On the other hand, PA policies are likely to affect current practitioner prescribing habits, perhaps due to a desire to avoid PA $[8,14]$, thus resulting in fewer and/ or lower-dose prescriptions. Those interviewed stated that they would be more likely to stop and think before writing a high-dose opioid prescription if a PA policy existed $[8,16]$. One study that examined the effects of a PA requirement on the prescribing of Cox-2 inhibitors found that PA caused prescribers to restrict 
Table 1 Summary of Findings.

\begin{tabular}{|c|c|c|c|c|c|c|c|}
\hline \multirow[b]{2}{*}{ Health Factor or Outcome } & \multirow[b]{2}{*}{ Literature review } & \multirow[b]{2}{*}{$\begin{array}{l}\text { Stakeholder } \\
\text { Perspectives }\end{array}$} & \multicolumn{4}{|c|}{ Based on Stakeholder input and Literature Review } & \multirow{2}{*}{$\begin{array}{r}\text { Literature } \\
\text { Certainty of } \\
\text { evidence }\end{array}$} \\
\hline & & & Direction & Magnitude & Likelihood & Distribution & \\
\hline Prescribing of opioids & Modest decrease & No change & Modestly Beneficial & Medium & Probable & High & Medium \\
\hline $\begin{array}{r}\text { Time and cost for } \\
\text { prescribers }\end{array}$ & Increase & Increase & Adverse & Medium & Probable & Low & High \\
\hline $\begin{array}{r}\text { Prescription opioid } \\
\text { consumption }\end{array}$ & Modest Decrease & Mixed & Modestly Beneficial & Medium & Probable & Medium & High \\
\hline $\begin{array}{l}\text { Patient anxiety and stress } \\
\text { due to prescription denial }\end{array}$ & $\mathrm{N} / \mathrm{A}$ & Increase & Adverse & Medium & Probable & High & N/A \\
\hline $\begin{array}{r}\text { Addiction to prescription } \\
\text { opioids }\end{array}$ & Decrease & Mixed & Modestly Beneficial & High & Possible & High & Low \\
\hline $\begin{array}{r}\text { Other substance abuse } \\
\text { (mainly heroin) }\end{array}$ & Increase & Increase & Modestly Adverse & High & Possible & Medium & High \\
\hline $\begin{array}{r}\text { Heroin-related } \\
\text { illness, overdose, and } \\
\text { homelessness }\end{array}$ & Increase & $\mathrm{N} / \mathrm{A}$ & Modestly Adverse & High & Possible & Medium & High \\
\hline $\begin{array}{r}\text { Heroin-related crime and } \\
\text { incarceration }\end{array}$ & Increase & Increase & Modestly Adverse & Medium & Possible & Medium & High \\
\hline
\end{tabular}

\section{Table Key}

1) Literature Review/Stakeholder Perspectives:

a) Increase: health outcome will increase

b) Mixed: there were mixed perspectives about the health outcome

c) Decrease: health outcome will decrease

d) No Change: health outcome will not change

e) Unknown: insufficient evidence available

f) N/A: data was not gathered from the source

2) Direction: Effect is beneficial or adverse.

a) Beneficial: there will be an increase in the health state

b) Adverse: there will be an decrease in the health state

c) Neutral: there will be no change in the health state

d) Unknown: health state is unmeasured or unknown

3) Magnitude: Expected size of the effect (number of affected people, expected frequency or prevalence of symptoms, illness or injury).
a) Low: the impact on health is minor and/or temporary and does not pose a hazard or benefit
b) Medium: the impact on health is detectable, reversible and/or poses a minor to moderate hazard/benefit
c) High: the impact on health is substantial, lasting and/or poses a major hazard/benefit
d) Unknown: the impact on health is unknown or poses an unknown hazard/benefit

4) Likelihood: Chance that the effect will occur.
a) Unlikely: the impact is not likely to occur
b) Possible: the impact is likely to occur on a regular basis
c) Probable: the impact will almost certainly occur and persist over time
d) Unknown

5) Distribution: Distribution of effects among vulnerable populations to delineate equity factors.
a) High: high impact on vulnerable population
b) Medium: medium impact on vulnerable population
c) Low: low impact on vulnerable population
d) Unknown: unknown impact on vulnerable population

6) Certainty of Evidence: level of confidence that the effects will occur based on literature review.
a) High: Evidence strongly supports the characterization conclusions
b) Medium: Evidence moderately supports the characterization conclusions
c) Low: Evidence nominally supports the characterization conclusions
d) Unknown: There is little or insufficient evidence to support the characterization conclusions
e) N/A: Literature was not examined for the health impact 


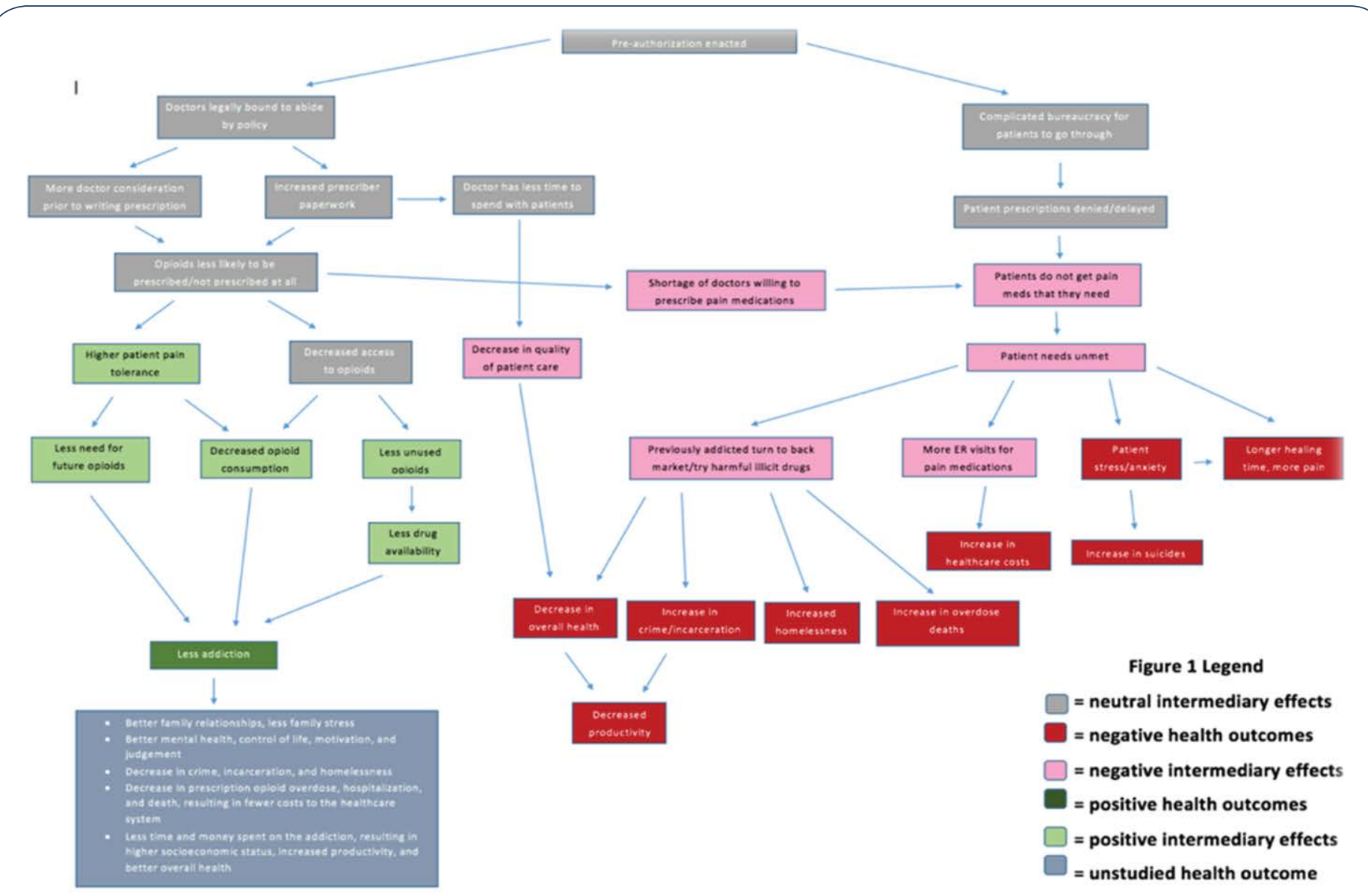

Figure 1 Casual pathway.

prescriptions to patients who truly needed them, while other patients were switched to less expensive alternatives that did not require PA [17]. Another study of Massachusetts' Medicaid program showed that after implementing PA to reduce high doses and daily doses of prescription opioids and to increase preferred therapeutic alternatives, the number of long-acting opioid analgesic users decreased by $17.8 \%$, and the number of claims decreased by $4.1 \%$ [18]. Another study found that PA was associated with an $8 \%$ decrease in long-acting oxycodone use [19]. Thus, requiring PA for prescription opioids could result in a modest decline in the number of opioid prescriptions written.

It would likely be beneficial for primary care physicians to cease writing high-dose long-acting opioid prescriptions, as primary care physicians prescribe opioids most frequently and are involved with patients who experience more opioid fatalities than other types of physicians [20]. However, a concern was expressed that if patients were unable to get opioids from their family doctor (or other general practitioner) due to the PA policy, they would to go to the emergency room to obtain them [10]. Alternatively, general practitioners may refer all such patients to pain specialists [8, 9]. Although this is not inherently a bad thing, as pain specialists are far better equipped to appropriately treat pain than general practitioners, specialist referrals and emergency room visits ultimately result in an increased financial burden to patients and the healthcare system [8]. Despite the negative impact of a PA policy on prescribers, particularly on pain specialists, it is likely that implementation of PA for high dose opioids will result in a modest reduction of opioid prescription rates.

An interview with a health economist revealed that those who are most likely to be influenced by policy decisions are those groups of individuals considered to be "elastic" (i.e., those that will change their behaviors due to policy enactment, changes in societal norms, price changes, etc.) [21]. Considering the key players of Utah's opioid epidemic, the most elastic groups are prescribers and patients that are not yet using prescription opioids. Those who are already addicted to prescriptions opioids would be considered inelastic. Given this, requiring PA for prescription opioids targets the right people, namely prescribers. Patients not using prescription opioids would also be an effective group to target with alternate policy approaches.

\section{Impacts to Pill Availability}

Changes to opioid diversion: Opioid diversion is a process that involves the transfer of opioids from a person to whom the drug was legally prescribed, to another person. Increased accessibility of prescription opioids has led to increased opioid diversion, which has contributed to increased opioid addiction. According to a study of opioid overdoses in Utah, $24 \%$ of opioids users were given drugs by friends or relatives, $18.2 \%$ were stolen, $16.4 \%$ were purchased from a friend, relative, or acquaintance, and $11 \%$ were purchased from a dealer [3]. Notably, a significant number of people who die from an opioid overdose do not have a prescription for the opioid that caused their death [22]. Should 
the proposed policy lead to a decrease in the amount of opioids prescribed, it can be assumed that there would be a decreased availability of unused drugs, and the problem of opioid diversion, and hence addiction, would be lessened [22].

In contrast to findings in the literature, several interviewed stakeholders gave a different opinion [8-10, 13]. Though many stakeholders felt that prescribing rates may slightly decrease, many thought that the resulting avoided addiction would be minimal. They also believed that there would be negligible decreases to other opioid-associated problems, such as opioid diversion, storage of unused drugs, and selling to the black market. Many felt that the added burden of PA was not worth the negligible amount of avoided opioid addictions. Additionally, many doctors felt that the mandatory controlled substances prescribing education required of all prescribers in Utah has already done much to reduce the number of opioid prescriptions, and that a PA policy is unlikely to further reduce prescribing rates and drug availability [8-10, 13].

Though the conclusions of stakeholders and literature differed, the HIA team gave more weight to scientific-based literature findings due to the greater potential bias of prescribers towards the inconvenience of PA; however, the recommendations will reflect consensus views of stakeholders. Thus, it was concluded that the proposed policy is likely to produce a modest decrease in addiction rates due a decrease in opioid availability.

\section{Impacts to Patients}

Changes to patient's physical and emotional health: PA has many effects on patients and their well-being. Firstly, PA causes patients to deal with more bureaucracy, which could delay or prevent them from getting necessary medication [8-10, 13, 23]. Physicians reported that PA may require multiple attempts before patients can get approval and obtain prescriptions that they need, delaying the process [9]. Additionally, patients may have to call or visit their doctor or pharmacy multiple times to obtain approval, which can be a strain to vulnerable patients, especially elderly or disabled patients who may have difficulty arranging transportation [24, 25]. Though it is possible for patients to bypass the PA denial by simply paying for their medications outof-pocket, the costs of opioid medications can range from $\$ 80$ to hundreds of dollars per prescription [25]. These costs would likely be too high for many patients to afford on their own, unfairly disadvantaging poor patients. In addition, PAs are overseen by insurance companies, not medical personnel, which could increase the likelihood of patient prescriptions being delayed or denied if the policy is enacted. The general opinion among the doctors interviewed was that PAs are not overseen by someone who can make appropriate, informed decisions, due to the fact that insurance companies know little about the patients or their diagnoses and treatment plans [9, 10, 12, 26].

Denied or delayed prescriptions can cause patients to feel anxious, stressed, angry, abandoned and emotionally hurt. Untreated patients in significant pain for long periods of time have more psychologically difficult recoveries and longer healing times [9]. In addition, multiple doctors stated that people who are addicted to opioids - people that really want them - will still find ways to get them even when PA is required $[9,10]$. However, the proposed policy could punish people who genuinely need opioid pain medications, causing all of the previously mentioned effects.

Changes in source of drugs Another serious health effect of the policy, mentioned by doctors, is the possibility that people who are already addicted to opioids will be less able to get prescription opioids, and that they will turn to the black market or illegal drugs to satisfy their addiction $[9,16]$. Both of these outcomes are more likely for younger patients [26]. Prescription drug users frequently move onto heroin, an illegal and powerful opioid, because it is much less expensive and often easier to get; studies have shown almost half of young heroin users start with abusing prescription opioids [27, 28]. A study reporting the effects of introducing an abuse-deterrent opioid showed that opioid dispensing dropped $19 \%$ and prescription opioid overdoses dropped 20\%; however, heroin overdose rose $23 \%$ as a result [29]. Reducing the supply of opioids without addressing their demand can result in people turning to heroin and other harmful drugs [23, 29].

Heroin users have other increased health risks in addition to the health risks experienced by prescription opioid abusers. For example, there is a higher risk of overdosing on heroin than prescription opioids because heroin is not always pure [2]. Heroin is injected, and many serious diseases such as HCV and HIV are spread through needle sharing [30]. Additionally, many heroin addicts are frequently homeless [31] or incarcerated [32, 33], both of which lead to additional negative health outcomes.

\section{Discussion}

\section{Recommendations}

The purpose of this study was to analyze the potential health impacts of the proposed opioid PA policy in Utah and to determine whether it would be an effective step in combatting Utah's opioid problem. After a thorough investigation of the proposed policy, it is anticipated that the policy will produce modest benefits, but may also complicate the process of filling opioid prescriptions for patients and their physicians, leading to several negative outcomes. In order to mitigate these negative health outcomes, we recommend that this policy be modified before moving forward. With the incorporation of this modification, it is recommended that the policy be adopted by the Utah State Legislature.

\section{Modification to the Proposed Policy}

Exempt pain specialists from the restrictions in the proposed policy: It is recommended that pain specialists be exempt from the PA policy, given that many of the prescriptions they write fall above the 90 morphine equivalent cut-off for the proposed PA, and that pain specialists have already undergone further training to deal with chronic pain and prescribe opioids appropriately. This would also help mitigate the significant increase in time and cost for pain specialists that is likely to result from this policy. In addition, maintaining the PA requirement for general practitioners will likely lead to increased patient referrals to pain specialists for patients with pain management issues. This will ensure that patients experiencing enough chronic pain to require high or chronic doses of prescription opioids receive appropriate care for their pain issues, and will minimize psychological stress associated with PA. 


\section{Alternative policy possibilities among elastic groups}

The following is a list of policy alternatives that would impact elastic groups involved in the opioid epidemic (i.e., doctors, patients, insurers). These policy alternatives are likely to further impact addiction rates without resulting in some of the negative effects associated with PA as identified in the HIA.

\section{Doctors as the target population}

1. Increased monitoring of the Controlled Substance Database (CSD): Monitoring of the CSD by qualified personnel from the Utah Medical Association or other credible institution to find harmful prescribing patterns and perform audits on implicated doctors is needed. Offending doctors could be reported to lawmakers, have a license suspended, or be required to undergo intensive education.

2. Additional doctor education: Yearly mandatory education by the Utah Medical Association is needed to arm physicians with accurate and up-to-date knowledge, allowing them to can make informed decisions regarding opioids, communicate better with patients, and set realistic treatment goals.

\section{Patients as the target population:}

1. Patient screening and contracts: Patients should undergo a thorough screening for need and substance abuse disorders prior to receiving an opioid prescription. Upon receiving an opioid prescription, patients should also be required to sign a contract with their physician stating that they will not go to another prescriber for an opioid prescription, that refills cannot be done over the phone, and that refills cannot be given before a certain date, among other clauses.

2. Patient education: Better patient education is needed to change patient expectations about pain and pain management. Patients need to be informed that opioids are not the first line of treatment for pain. In addition, patients need to understand the consequences of sharing or selling their unused prescription opioids, and need to learn how to properly use medications and dispose of unused medications.

\section{Other Alternative Policy Suggestions}

1. Insurance coverage of alternative methods of pain management: Adequate coverage/reimbursement by insurance companies for alternative treatment options, such as specialist care, physical and cognitive therapy, and other self-regulatory pain management methods is needed to provide a comprehensive approach to pain management to help patients better manage pain.

2. Expand drug addiction rehabilitation centers, staff, and resources: Expanding the resources of drug rehabilitation and detox centers for opioid abuse patients could help decrease the number of opioid addicts and prevent relapse by addicts.

\section{Limitations}

Despite best efforts by the research team to conduct a comprehensive assessment, this assessment has some limitations. First, there are insufficient Utah-specific data concerning the health care costs and work productivity costs of opioid abuse. This made it difficult to predict changes in costs due to changes in addiction. Second, a lack of data concerning the effect of PA on patient care and outcomes led the HIA team to gather this information from stakeholder interviews. Third, this HIA did not employ random selection of stakeholders for data collection. Thus, stakeholder input may not be fully representative. Stakeholders were selected to include a variety of medical specialists, but were based on existing connections with the HIA team, their accessibility in being contacted, and their willingness to participate in the HIA process. Fourth, not all selected stakeholders responded. Notably, opinions from affected insurers and key representatives from the Utah State Health Department were unable to be collected as part of this HIA. Fifth, there were discrepancies between findings among stakeholders, and between stakeholders and gathered literature. The HIA team alleviated these problems by seeking numerous and varied stakeholder opinions, and by using the best possible judgement when framing the recommendations for the proposed policy. Sixth, the first four HIA phases are reported in this paper since, at the time of writing, monitoring and evaluation phases continue beyond the time when lawmakers consider the proposed bill during the legislation session (January through March 2017). Further, the first four phases have the greatest worth to decision makers and academic audiences because the HIA results and recommendations are valued. Further, though somewhat beneficial to academic audiences, the last two phases are of greatest benefit to HIA administrators.

\section{Conclusion}

This HIA provides a valuable contribution to the fight against opioid addiction in Utah by providing a thorough analysis of a policy that would require PA for high doses of opioid prescriptions, coprescription of opioids with benzodiazepines, and new patients starting long-acting opioids. With modification, this policy could provide a modest decrease to the rate of opioid addiction in Utah. Given this, it is recommended that the Utah State Legislature move forward with this policy following implementation of the recommended modification. Further, additional policies for consideration should include those that affect groups with the highest elasticity (likelihood for change).

\section{Acknowledgement}

Ms. Chandni Murali contributed to a draft HIA report and assisted with the literature review and stakeholder interviews 


\section{References}

1 (2014) Utah Department of Health. Prescription Drug Overdoses.

2 Volkow ND (2014) Prescription Opioid and Heroin Abuse. House Committee on Energy and Commerce Subcommittee on Oversight and Investigations. National Institute on Drug Abuse.

3 Johnson EM, Lanier WA, Merrill RM, Crook J, Porucznik CA, et al. (2013) Unintentional prescription opioid-related overdose deaths: description of decedents by next of kin or best contact, Utah, 20082009. Journal of general internal medicine 28: 522-529.

4 Ward R (2016) Reversing the opioid epidemic by decreasing high risk opioid prescribing habits. Utah Health and Human Services Interim Committee.

5 (2016) State of Utah. Insurance Coverage for Opioids.

6 Leonard W (2016) Utah lawmaker introduces legislation to curb opioid epidemic. Deseret News.

7 (2016) Centers for Disease Control and Prevention. Health Impact Assessment.

8 (2016) Interview with a Medical Director/General practice physician.

9 (2016) Interview with a family practice physician.

10 (2016) Interview with a colorectal surgeon.

11 (2016) Interview with a pharmacist from a large, corporate pharmacy.

12 (2016) Interview with a dentist.

13 (2016) Interview with a physiatrist/pain specialist. 2016.

14 Brown CM, Richards K, Rascati KL, Gavaza P, Corbell Z, et al. (2008) Effects of a psychotherapeutic drug prior authorization (PA) requirement on patients and providers: a providers' perspective. Administration And Policy In Mental Health 35: 181-188.

15 Morley CP, Badolato DJ, Hickner J, Epling JW (2013) The impact of prior authorization requirements on primary care physicians' offices: report of two parallel network studies. Journal of The American Board Of Family Medicine: JABFM. 26: 93-95.

16 (2016) Interview with Representative Raymond Ward, Legislative Sponsor of the proposed policy.

17 Siracuse MV, Vuchetich PJ (2008) Impact of Medicaid prior authorization requirement for COX-2 inhibitor drugs in Nebraska. Health services research 43: 435-450.

18 Garcia MM, Angelini MC, Thomas T, Lenz K, Jeffrey P, et al. (2014) Implementation of an opioid management initiative by a state Medicaid program. Journal of managed care \& specialty pharmacy 20: 447-454.
19 Morden NE, Zerzan JT, Rue TC, Heagerty PJ, Roughead EE, et al. (2008) Medicaid prior authorization and controlled-release oxycodone. Medical care 46: 573-580.

20 Porucznik CA, Johnson EM, Rolfs RT, Sauer BC (2014) Specialty of prescribers associated with prescription opioid fatalities in Utah, 2002-2010. Pain medicine 15: 73-78.

21 (2016) Interview with a health economist.

22 Meyer R, Patel AM, Rattana SK, Quock TP, Mody SH, et al. (2014) Prescription Opioid Abuse: A Literature Review of the Clinical and Economic Burden in the United States. Population Health Management 17: 372-387.

23 (2016) Interview with a member of a county health department representative.

24 Henry J Kaiser (2003) Prior authorization for Medicaid presctiptions drugs in five states: Lessons or policy makers. Kaiser Commision on Medicaid and the Uninsured.

25 (2016) Interview with a pharmacist from a private pharmacy.

26 (2016) Interview with an addictions counselor.

27 Cicero TJ, Ellis MS, Surratt HL, Kurtz SP (2014) The changing face of heroin use in the united states: A retrospective analysis of the past 50 years. JAMA Psychiatry 71: 821-826.

28 (2014) National Institute on Drug Abuse. Heroin: How is heroin linked to prescription drug abuse?

29 Larochelle MR, Zhang F, Ross-Degnan D, Wharam J (2015) Rates of opioid dispensing and overdose after introduction of abusedeterrent extended-release oxycodone and withdrawal of propoxyphene. JAMA Internal Medicine. 175: 978-987.

30 (2014) National Institute on Drug Abuse. Heroin: Why does heroin use create special risk for contracting HIV/AIDS and hepatitis B and C?

31 Mutlu E, Alaei A, Tracy M, Waye K, Cetin MK (2016) Correlates of injection drug use among individuals admitted to public and private drug treatment facilities in Turkey. Drug and alcohol dependence 164: 71-81.

32 Fox AD, Maradiaga J, Weiss L, Sanchez J, Starrels JL, et al. (2015) Release from incarceration, relapse to opioid use and the potential for buprenorphine maintenance treatment: a qualitative study of the perceptions of former inmates with opioid use disorder. Addict Sci Clin Pract 10: 2.

33 Sutherland R, Sindicich N, Barrett E, Whittaker E, Peacock A, et al. (2015) Motivations, substance use and other correlates amongst property and violent offenders who regularly inject drugs. Addictive Behaviors 45: 207-213. 